\title{
BATTERY MANAGEMENT SYSTEM FOR AN ELECTRIC VEHICLE
}

\author{
F. Issa \\ Valahia University of Targoviste, Faculty of Electrical Engineering, Electronics and Information Technology \\ E-mail: fadelissa@yahoo.com
}

\begin{abstract}
In the electric vehicle industry, a battery management system is needed when voltages are in the value of hundreds of volts. The battery management system is not only used to estimate the remaining capacity of the batteries but also to prevent various hazards that may occur in case of improper use. The paper offers a review of the battery management system that is used in the modern electric vehicles. The system is described along with its functions including the practical methods for balancing the battery and the type of batteries used in the automotive industry.
\end{abstract}

Keywords: BMS, baterry, electric vehicles

\section{INTRODUCTION}

The basic meaning for electric vehicles is any vehicle powered by a source of electricity. In addition to other definitions provided, the electric vehicle is a system that has as energy source an accumulator/battery that has the ability to be charged by using one or more electric motors of the vehicle [2]. The system used for the electric drive consists of:

- High voltage battery that is equipped with a management system for regulation and charging.

- Electric motor/generator together with a system for control and cooling.

- Transmission.

- The system used for braking.

- Air conditioning that uses high voltage.

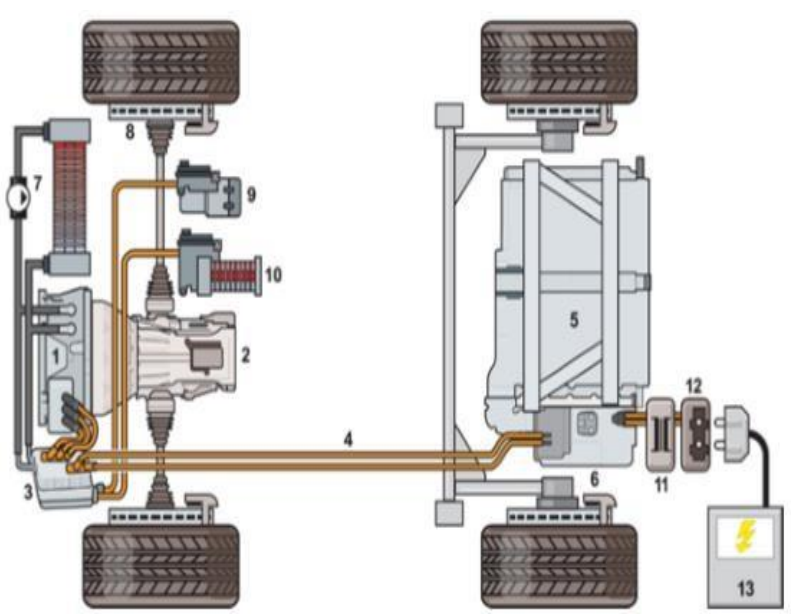

Figure 1. Electrical Vehicle Components.
The main components of electric vehicles are illustrated in Figure 1 and they form the entire propulsion and power system. These components are as follows:
1) Electric motor
2) Transmision
3) Power electronics
4) High voltage cables
5) High voltage battery
6) Battery management electronics
7) Cooling system
8) Brakes
9) High voltage compresor
10) High voltage heating
11) Charger
12) Charging connector
13) External power source

\section{BATTERY MANAGEMENT SYSTEM}

The system used for battery management (BMS) is generally installed in the dashboard of electric cars. Its function is to connect the vehicle to the battery and its role is vital.[1] As battery management functions, they include optimizing battery performance and optimizing the vehicle for a reliable and safe operation. For electric vehicles, the development of a comprehensive and mature battery management system is crucial, as it is similar to the engine management system in an internal combustion engine. [4] The necessary indicators for a battery management system include the safety status, use, optimization, performance and life of the battery to reduce the possibility of starting an ignition when it is overcharged or reducing the capacity of the cells when it is discharged repeatedly. Also, the monitoring and control of the electronic components that deal with the battery packs that are found in their management system is a very important objective.[3] When the management system detects certain anomalies in the system such as overvoltage or overheating, it will send a notification to the user and will automatically correct the problem by a preset method. In order to streamline the electricity consumption and the way of communicating with the other components of the vehicle, the system must also keep in mind the temperature level of the system. This system must have the following functions:

- Data collection

- Protection

- Determination of capacity and battery condition

- Charge control and battery discharge 
- Balancing the batteries

- Thermal management

- Display battery status

- Communication with all battery components

A common battery management system is made from a number of functional components. These components include field effect transmitters (FET), fuel indicator (in our case the battery charge percentage), cell monitoring and balance, temperature monitoring and real-time clock [5].

a) Field effect transmitters (FET): This component is used to connect or disconnect the vehicle's battery when it is in use or in charging mode. This behavior is based on real-time measurements of the vehicle's battery. Measurements are made for voltage, current and circuits.

b) Fuel indicator: This component has the role of displaying the battery charge level both during charging and during use. The load level is calculated by multiplying the current variable and the time variable.

c) Cell monitoring and balance: To find out the health of the battery it is necessary to monitor the voltage of each cell that is found in a battery pack. Each cell has a standard voltage range that must be found and maintained during both in charging and discharging mode. For example, for a common Li-Ion battery cell that is used in the electric vehicle industry, the range is between $2.5 \mathrm{~V}$ and $4.2 \mathrm{~V}$. This voltage range is given by the battery chemistry. If the voltage range is not respected, the battery degrades and the life is significantly reduced which will make the cell unusable.

d) Temperature monitoring: Batteries used in the electric vehicle industry provide a fairly high amount of current at a constant voltage and this leads to battery heating. If the heating of the battery is not controlled it can catch fire and explode due to the chemicals used which are also very volatile. If the battery is damaged by impact or puncture, this will cause it to ignite due to chemicals coming into contact with oxygen. Temperature monitoring is not only used as a safety measure but is also used to charge or discharge the battery. Each cell pack is monitored separately by thermistors that have an internal reference voltage using an analog-to-digital converter to reduce the reading error of the temperature compared to the external environment.

\section{BATTERY BALANCING METHODS}

For an ideal battery management system that would not need balancing, all cells would need to have the same voltage, internal resistance, aging degree and similar connection mode. But the properties of each cell differ and this difference comes from the manufacturing process. These differences in properties can lead to considerable imbalances over time resulting in reduced battery life. Due to the way these batteries are built being often spot welded it makes it impossible to change defective cells requiring replacement of the entire battery. To optimize a similar system requires a process of measuring the parameters of each cell but involves a high cost for the battery manufacturer [6].

In order to allow the communications between the components of the vehicle management system, the CAN bus protocol is generally used, this protocol is already used in classic vehicles and is also adopted in electric vehicles.

Different methods are used to balance the batteries, below are explained two methods being the most practical.

\section{1) First method: Balancing by individual cell loading}

To balance or charge an individual cell it is necessary to apply a charging algorithm that uses a constant current and voltage individually for each cell. It requires the presence of voltage sources that have a floating mass (reference), which requires a galvanically isolated supply for each cell to the rest of the cells and to the general source. This is a disadvantage that leads to impracticality in modules with more than 3 cells, and the galvanic insulation mentioned above limits the maximum charging current. Thus, each cell is optimally charged resulting in a charge independent of the other cells (Figure 2). By using this method, the capacity of each cell can be carefully monitored and premature wear or overheating can be determined.

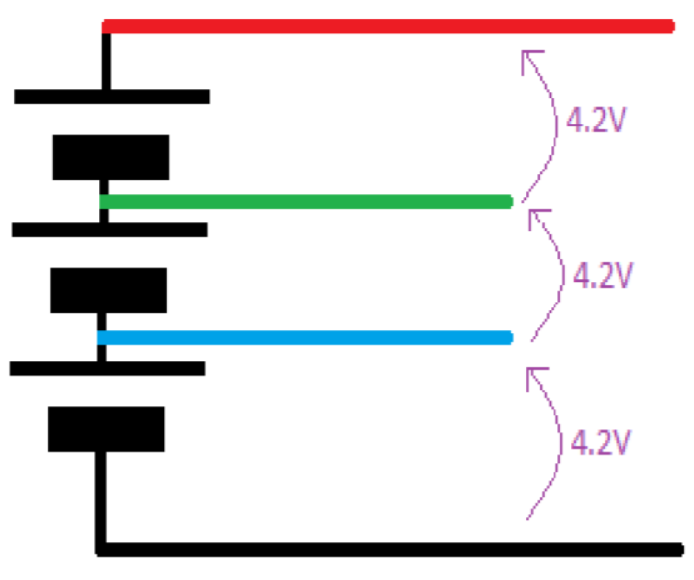

Figure 2. Individual cell loading.

2) Second method: Balancing by individual cell discharge To balance the battery by individually discharging the cells it is necessary to apply a charging voltage to the ends of the battery (power heads) and the excess power will be discharged using resistors in parallel with the battery cells (Figure 3). Therefore, if one cell charges faster than the 
other (if it has a smaller capacity than the other cells), it connects a load in parallel until the voltage drops and is similar to the cell voltage.

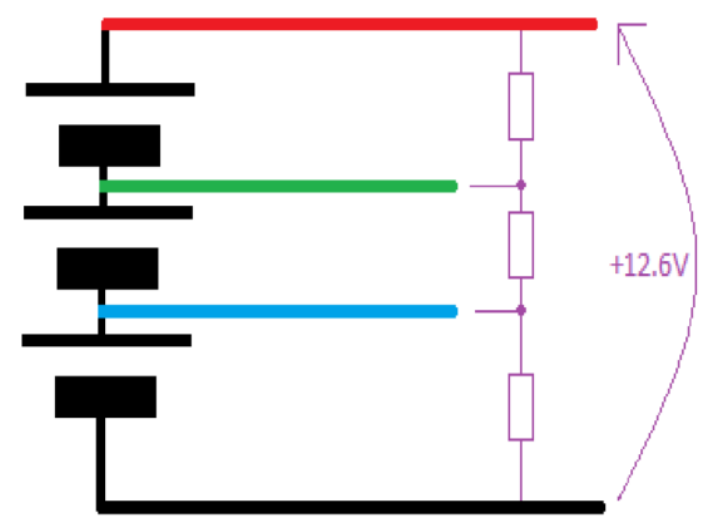

Figure 2. Individual cell discharge.

\section{TYPES OF BATTERIES USED FOR ELECTRIC VEHICLES}

The most common source of energy storage for electric vehicles is batteries. The mode of operation of these batteries is given by the chemical reactions that take place during discharge or charging. Chemical reactions gradually reduce the capacity of the batteries by degrading the chemical structure until the battery becomes unusable. This battery degradation can be delayed by the way the battery is used. For example, subjecting batteries to extreme temperatures during charging or use results in a shorter lifespan than those that operate under normal temperature conditions. The safety of the batteries is given by the operation in the parameters offered by the manufacturer and any deviation from the limits given by it can represent a danger for the user. For the safety of the user and a safe operation of these batteries, the conditions specified by the manufacturer must be observed, thus maintaining the limits of the charging and discharging currents, voltage and temperature.

Monitoring the battery voltage does not involve monitoring their remaining capacity. The energy left in the battery depends on the state of charge (SOC) being an essential parameter for monitoring the remaining capacity in the battery. The current capacity of the battery divided by the nominal capacity defines the SOC parameter. Monitoring the SOC parameter is essential to avoid battery failure in overload conditions without indicating the remaining battery life.

The use of batteries for electric vehicles depends on their type depending on the requirements and conditions in which they are operated. Most internal combustion vehicles use a Pb-Acid battery, these batteries have a stable chemical composition but their weight is high and are often used in stationary electricity storage systems.
Li-Ion, Li-Polymer or LiFePo4 batteries are commonly used to provide electricity for electric vehicles. These batteries have an unstable chemical composition and improper use leads to overheating and explosion.

\section{1) Lithium-Iron phosphate batteries (LiFePo4)}

This type of rechargeable battery uses $\mathrm{LiFePo} 4$ for the cathode and for the anode uses a carbon electrode that has a metal collector. Thus, being ideal to be used in electric vehicles for the high energy density $/ \mathrm{kg}$. It is worth mentioning that these batteries withstand a large temperature range, have a long life and an increased resistance to overheating and explosions.

Characteristics:

- $\quad$ Specific energy: 90-110Wh / kg

- Energy density: 220Wh / L

- Lifespan: 2000 cycles

- Rated voltage: $3 \mathrm{~V}-3.3 \mathrm{~V}$

- Maximum voltage: $3.65 \mathrm{~V}$

\section{2) Li-Polymer batteries (Li-Poli)}

This type of rechargeable battery is derived from Li-Ion batteries and a polymer is used for the electrolyte. The specific energy is higher than other batteries with a similar chemical composition and its usage is vast covering mobile devices, laptops and electric vehicles.

Characteristics:

- $\quad$ Specific energy: 100-265Wh / kg

- Energy density: 250-750Wh / L

- Lifespan: 1000 cycles

- $\quad$ Rated voltage: $3.7 \mathrm{~V}-3.85 \mathrm{~V}$

- Maximum voltage: $4.2 \mathrm{~V}-4.4 \mathrm{~V}$

3) Lithium-Ion batteries (Li-Ion)

This type of rechargeable battery is one of the most used in the electric vehicle industry for its high energy density, stable chemical composition and for economic reasons. An important feature of these batteries is that they keep their balance from the factory. A battery pack consisting of such cells requires little maintenance, it should be noted that the batteries are equipped with an overpressure valve being found in the upper part on the positive terminal and the role of this valve is to mechanically and electrically disconnect the cell from the rest of the cells, if pressure is accumulated in these kinds of cells.

Characteristics:

- Specific energy: 00-265Wh / kg

- Energy density: 250-680Wh / L

- Lifespan: 1000 cycles

- $\quad$ Rated voltage: $3.6 \mathrm{~V}-3.85 \mathrm{~V}$

- Maximum voltage: $4.1 \mathrm{~V}-4.4 \mathrm{~V}$ 


\section{CONCLUSIONS}

Battery management systems are specifically designed only for a certain type of battery that has a known number of serial cells and this involves high production costs. Even though the system is modular, it is not designed to accommodate a wide variety of serial cells. The battery management system ensures the proper functioning of the entire battery in charging or discharging conditions by monitoring its voltage, current and temperature. It is worth mentioning that the system is able to prepare the battery for a more intense use or for a faster charge by changing its temperature, voltage and current.

\section{REFERENCES}

[1] F. Vellucci, V. Sglavo, G. Pede, Life cycles test on a lithium battery system, IECON 2014, November 2014.

[2] Chuan Sheng Si, Development research about the power battery management system of pure electric vehicle, 2011 International Conference on Consumer Electronics, Communications and Networks (CECNet), April 2011.

[3] B.P. Divakar, K.W.E. Cheng, H.J. Wu, Battery management system and control strategy for hybrid and electric vehicle, 2009 3rd International Conference on Power Electronics Systems and Applications (PESA), May 2009.

[4] Dongping Xu, Lifang Wang, Jian Yang, Research on Li-ion Battery Management System, 2010 International Conference on Electrical and Control Engineering, June 2010.

[5] Hardik Keshan, Jesse Thornburg, Taha Selim Ustun, Comparison of lead-acid and lithium-ion batteries for stationary storage in off-grid energy systems, 4th IET Clean Energy and Technology Conference (CEAT 2016), November 2016.

[6] Siguang Li, Chengning Zhang, Study on Battery Management System and Lithium-ion Battery, 2009 International Conference on Computer and Automation Engineering, March 2009. 